\title{
ON MODULAR COMPLEMENTED AND ANNIHILATOR ALGEBRAS
}

\author{
T. HUSAIN ${ }^{1}$ AND PAK-KEN WONG ${ }^{2}$
}

\begin{abstract}
Let $\boldsymbol{A}$ be a modular complemented algebra. We give some necessary and sufficient conditions for some special classes of algebras $A$ to be annihilator or dual algebras.
\end{abstract}

1. Introduction. Modular complemented algebras were introduced by Yood in [7], where he developed a structure and ideal theory for these algebras. In this paper our aim is to investigate the relationship among modular complemented, annihilator and dual algebras.

Let $A$ be a modular complemented algebra. We show that if $I+I^{\perp}=A$ for all minimal (left, right) ideals $I$ of $A$, then $A$ is an annihilator algebra if and only if every nonzero left ideal of $A$ contains an element with right adjoint. If $J+J^{\perp}=A$ for all (left, right) ideals $J$ of $A$, and $A$ is a left or right annihilator algebra, then it is a dual algebra.

2. Notation and preliminaries. Let $A$ be an algebra over the complex field $C$ which is a pre-Hilbert space in the inner product $(x, y) . A$ is called a right-modular complemented algebra if it satisfies the following conditions:

(a) the product $x y$ is continuous in each variable separately,

(b) every right or left ideal $I$ for which $I^{\perp}=(0)$ is dense in $A$,

(c) the intersection of all closed modular maximal right ideals is (0) and $M^{\perp}$ is a right ideal for each closed modular maximal right ideal $M$.

REMARK. From (c) it follows that every right-modular complemented algebra is semisimple.

Similarly we define a left-modular complemented algebra. It has been shown [7] that an algebra $A$ is right-modular complemented if and only

Received by the editors July 19, 1971.

AMS 1969 subject classifications. Primary 4650; Secondary 4655.

Key words and phrases. Modular complemented algebra, annihilator algebra, dual algebra, Hilbert algebra, proper $H^{*}$-algebra.

${ }^{1}$ This work was supported by a N.R.C. grant.

2 The second author was supported by a postdoctoral fellowship at McMaster University.

(c) American Mathematical Society 1972 
if it is left-modular complemented. In this case, $K^{\perp}$ is a right (left) ideal for any right (left) ideal $K$ of $A$ (see [7, p. 262, Theorem 2.1]).

Let $A$ be an algebra which is a pre-Hilbert space. As in [7], we say that $u^{l}$ is a left adjoint for $u$ if $(u x, y)=\left(x, u^{l} y\right)$ for all $x, y \in A$. If $A$ is semisimple, then the left adjoint is unique if it exists. Let $u, v \in A$. If $u^{l}, v^{l}$ exists then $(u v)^{l}$ exists and $(u v)^{l}=v^{l} u^{l}$. If $u=u^{l}$, then $u$ is said to be left selfadjoint. Similarly $u^{r}$ is called the right adjoint if $(x u, y)=\left(x, y u^{r}\right)$ for all $x, y \in A$.

For any subset $E$ in an algebra $A$, let $L(E)(R(E))$ denote the left (right) annihilator of $E$ in $A$. Suppose $A$ is a topological algebra then we call $A$ an annihilator algebra provided that, for every closed left ideal $M$ and for every closed right ideal $N$, we have $R(M)=(0)$ if and only if $M=A$ and $L(N)=(0)$ if and only if $N=A$. If $M=L(R(M))$ and $N=R(L(N))$, then $A$ is called a dual algebra.

In this paper, all algebras and linear spaces are over the complex field $C$. The results of this paper heavily depend on [6] and [7].

3. Characterizations of annihilator and dual algebras. It is known that a modular complemented algebra need not be an annihilator algebra (see [7, p. 263]). In our first result, we give a characterization for annihilator modular complemented algebras.

Let $A$ be a topological algebra. Then $A$ is called a left annihilator algebra if for every closed right ideal $M$ of $A, L(M) \neq(0)$ and $L(A)=(0)$. Similarly we define a right annihilator algebra.

THEOREM 3.1. Let $A$ be a modular complemented algebra such that $I \oplus I^{\perp}=A$ for all minimal (left, right) ideals $I$ of $A$. Then the following statements are equivalent:

(i) $A$ is an annihilator algebra.

(ii) $A$ is a right annihilator algebra.

(iii) Every element of the socle $S$ of $A$ has a right adjoint.

(iv) Every nonzero left ideal of $A$ contains an element with right adjoint.

Proof. (i) $\Rightarrow$ (ii). This is clear.

(ii) $\Rightarrow$ (iii). Suppose $A$ is a right annihilator algebra. Let $I$ be a minimal left ideal of $A$. Since $A=I \oplus I^{\perp}$, it follows easily that $I^{\perp}$ is a maximal closed left ideal. Thus by $\left[1\right.$, p. 568 , Theorem 3.4], $I^{\perp}$ is a modular maximal left ideal. Hence by the proof of $[6$, p. 44, Theorem 4.5] each element of $I$ has a right adjoint and so does $S$.

(iii) $\Rightarrow$ (iv). Let $M$ be a nonzero left ideal of $A$. Since $S$ is dense in $A$ (see the proof of [7, p. 262, Theorem 2.1]), we have $S \cap M \neq(0)$. Therefore (iv) follows from (iii). 
(iv) $\Rightarrow$ (ii). Assume (iv). Let $T$ be the set given in the proof of [7, p. 262, Theorem 2.1]. Then $T$ is a dense right ideal of $A$ and each element in $T$ has a left adjoint. Let $J$ be a proper closed left ideal of $A$. Then $J^{\perp} \neq(0)$. Let $x$ be an element in $J^{\perp}$ which has a right adjoint $x^{r}$. Let $z \in J$ and $y \in T$. Then $(y x, z)=\left(x, y^{l} z\right)=0$. Since $T$ is dense in $A$, we have $(A x, z)=$ (0) and so $\left(A, z x^{r}\right)=(0)$ which gives $z x^{r}=0$. Hence $J x^{r}=(0)$ and so $R(J) \neq(0)$. Therefore $A$ is a right annihilator algebra.

(ii) $\Rightarrow$ (i). Suppose (ii) holds. Let $I$ be a minimal left ideal of $A$. By the argument in (ii) $\Rightarrow$ (iii), $I^{\perp}$ is a modular maximal left ideal. Hence by the proof of [7, p. 263, Theorem 2.3], each element of $I$ has a left adjoint and so does $S$. Hence by a similar argument in the proof (iv) $\Rightarrow$ (ii), we can show that $A$ is a left annihilator algebra. This completes the proof of the theorem.

REMARK. A similar form of Theorem 3.1 holds for left annihilator algebras.

THEOREM 3.2. Let $A$ be a modular complemented algebra such that $J \oplus J^{\perp}=A$ for all closed (left, right) ideals $J$ of $A$. Then the following statements are equivalent:

(i) $A$ is a dual algebra.

(ii) $A$ is a left or right annihilator algebra.

Proof. (i) $\Rightarrow$ (ii). This is clear. (ii) $\Rightarrow$ (i). Assume (ii). Then by Theorem 3.1 and its remark, $A$ is an annihilator algebra. Let $I$ be a proper closed right ideal of $A$. Since $I^{\perp} \neq(0)$ and since the socle of $A$ is dense in $A$, it follows that $I^{\perp}$ contains a minimal right ideal $J$. By the proof of Theorem $3.1, J^{\perp}$ is a closed modular maximal right ideal and clearly $J^{\perp} \supset I$. Let $\left\{M_{\lambda}: \lambda \in \Lambda\right\}$ be the family of all closed modular maximal right ideals of $A$ containing $I$. Then $\left\{M_{\lambda}: \lambda \in \Lambda\right\}$ is not empty. Let $M=\bigcap\left\{M_{\lambda}: \lambda \in \Lambda\right\}$. We claim that $M=I$. In fact, suppose this is not so. Then there exists an element $x \in M$ such that $x \notin I$. Since $I \oplus I^{\perp}=A$, we can write $x=x_{1}+x_{2}$ with $x_{1} \in I$ and $x_{2} \in I^{\perp}$. Hence $x_{2}=x-x_{1} \in I^{\perp} \cap M$ and so $I^{\perp} \cap M$ contains a minimal right ideal $J$ whose orthogonal complement $J^{\perp}$ is a closed modular maximal right ideal by the above argument. Therefore by the proof of $\left[6\right.$, p. 44, Theorem 4.5], $J$, and hence $I^{\perp} \cap M$, contains a left selfadjoint minimal idempotent $e$. Since $e A \subset I^{\perp}$, we have $(1-e) A=$ $(e A)^{\perp} \supset I$. Therefore $(1-e) A \supset M$. But $e \in M$, a contradiction. Hence $M=I$. Since each $M_{\lambda}=R\left(L\left(M_{\lambda}\right)\right)$, we have $I=R(L(I))$. Similarly we can show that $N=L(R(N))$ for all closed left ideals $N$ of $A$. Therefore $A$ is a dual algebra. This completes the proof.

A modular complemented algebra is said to be complete if it is complete in its inner product norm. 
COROLlary 3.3. Let $A$ be a complete modular complemented algebra. Then the conditions (i), (ii), (iii) and (iv) in Theorem 3.1 are equivalent to the statement that $A$ is a dual algebra.

Proof. This follows from Theorems 3.1 and 3.2.

RemarK. It is well known that a proper $H^{*}$-algebra $A$ is dual. This result also follows from Corollary 3.3, since every element of $A$ has left and right adjoints.

If a modular complemented algebra is a normed algebra under its inner product norm, then we say that it is a normed modular complemented algebra.

THEOREM 3.4. Let $A$ be a complete normed modular complemented algebra. Then

(i) $A$ is a dense left ideal of a dual $B^{*}$-algebra $B$.

(ii) If $A$ is also a right ideal of $B$, then $A$ is a dual algebra.

Proof. (i) For each $x \in A$, let $U_{x}$ be the operation of left multiplication by $x$ and $\left|U_{x}\right|$ the operator bound of $U_{x}$. Let $B$ be the closure of $\left\{U_{x}: x \in A\right\}$ in the uniform topology. Since $A$ is semisimple, the mapping $x \rightarrow U_{x}$ is one-one. Therefore we can identify $A$ as a subalgebra of $B$. Let $T$ be the algebraic sum of the $M^{\perp}$ as $M$ ranges over the set of all closed modular maximal right ideals of $A$. By the proof of [6, p. 44, Theorem 4.5], $T$ is dense in $A$, the left adjoint $a^{l}$ exists for all $a \in T$ and $a^{l} \in T$. Clearly $U_{a}^{l}=\left(U_{a}\right)^{*}$ and so $T^{*}=T$ in $B$. Since $T$ is dense in $B$, it follows that $B=B^{*}$. Therefore $B$ is a $B^{*}$-algebra. Since the socle of $B$ contains $T$, it follows from $[4$, p. 222 , Theorem 2.1] that $B$ is a dual algebra. For all $x$, $y \in A$, we have $\|x y\| \leqq|x|\|y\|$. This shows that $A$ is a left ideal of $B$ and completes the proof of (i).

(ii) Now it follows from (i) that $A$ is a dense two-sided ideal of $B$. For example, a proper $H^{*}$-algebra has such a property. Let $M$ be a nonzero right ideal of $A$. Then $M$ contains a minimal idempotent $e$ of $A$. It is clear that $e B=e A$ and so $e A$ is a minimal right ideal of $B$. Hence by [5, p. 261, Lemma (4.10.1)], $e A$ contains a hermitian minimal idempotent $f \in B$. It is easy to see that $f \in A$ and $f$ is a left selfadjoint element in $A$. Since $f \in M$, it follows from Corollary 3.3 that $A$ is a dual algebra.

4. Hilbert algebras. A complex algebra $A$ is called a Hilbert algebra (see [3]) if it is a pre-Hilbert space with an involution $x \rightarrow x^{*}$ having the following properties:

(a) $(x y, z)=\left(y, x^{*} z\right)$ for all $x, y, z \in A$,

(b) $(x, y)=\left(y^{*}, x^{*}\right)$ for all $x, y \in A$, 
(c) the operation of left multiplication $y \rightarrow x y$ is continuous on $A$ for each $x \in A$,

(d) the set of elements of the form $x y$ is dense in $A$.

It follows easily from (a) and (b) that $(y x, z)=\left(y, z x^{*}\right)$ for all $x, y, z \in A$. It is easy to see that the right multiplication is also continuous. Since $\|x\|=\left\|x^{*}\right\|$, the involution is continuous.

A net $\left\{e_{\alpha}\right\}$ in a normed algebra $A$ is called an approximate identity if it is bounded and $x=\lim _{\alpha} x e_{\alpha}=\lim _{\alpha} e_{\alpha} x$ for all $x \in A$.

The following result was proved by Yood for $B^{*}$-algebras (see [7, p. 267, Theorem 3.1]).

THEOREM 4.1. Let $A$ be an infinite-dimensional Banach *-algebra with an approximate identity $\left\{e_{\alpha}\right\}$. Then there does not exist an inner product for $A$ under which $A$ becomes a pre- $H^{*}$-algebra with the same involution.

Proof. Suppose otherwise. Let $|x|=(x, x)^{1 / 2}$ be the inner product norm and let $H$ be the completion of $A$ in the norm $|x|$. By the proof of [7, p. 267, Theorem 3.1], $H$ is a proper $H^{*}$-algebra and, for all $x \in A$, $|x| \leqq k\|x\|$, where $k$ is a constant. Now it is easy to see that $\left\{e_{\alpha}\right\}$ is also an approximate identity in $H$. Since $H$ is reflexive, by $[2$, p. 855 , Lemma 3.8], $H$ has an identity. Therefore by [7, p. 266, Corollary 2.7], $H$ is finite dimensional and so is $A$, a contradiction. This completes the proof.

TheOREM 4.2. Let $A$ be a Hilbert algebra. Then $A$ is a dual algebra if and only if $I=I^{\perp \perp}$ for all closed right ideals $I$ of $A$.

Proof. By the proof of [7, p. 268, Lemma 3.2], $x \in x A \cap A x$. Therefore $L(A)=R(A)=(0)$. Suppose $A$ is a dual algebra. Let $I$ be a closed right ideal of $A$. Then by the proof of [7, p. 268, Lemma 3.2], we have

$$
I=R(L(I))=\left(\left(I^{\perp}\right)^{* *}\right)^{\perp}=I^{\perp \perp} .
$$

Conversely suppose $I=I^{\perp \perp}$ for all closed right ideals $I$ of $A$. It is easy to see that $I^{\perp \perp}=R(L(I))=I$. Let $J$ be a closed right ideal of $A$. Hence $J^{*}=\left(J^{*}\right)^{\perp \perp}$ and so $J=J^{\perp \perp}$. It is now easy to see that $J=L(R(J))$. Therefore $A$ is a dual algebra.

Corollary 4.3. Let $A$ be a Hilbert algebra. If $I \oplus I^{\perp}=A$ for all closed right ideals I of $A$, then $A$ is a dual algebra.

\section{REFERENCES}

1. B. A. Barnes, Modular annihilator algebras, Canad. J. Math. 18 (1966), 566-578. MR 33 \#2681.

2. P. Civin and B. Yood, The second conjugate space of a Banach algebra as an algebra, Pacific J. Math. 11 (1961), 847-870. MR 26 \#622. 
3. J. Dixmier, Les algèbres d'opérateurs dans l'espace Hilbertien (Algèbres de von Neumann), Gauthier-Villars, Paris, 1969.

4. I. Kaplansky, The structure of certain operator algebras, Trans. Amer. Math. Soc. 70 (1951), 219-255. MR 13, 48.

5. C. E. Rickart, General theory of Banach algebras, University Series in Higher Math., Van Nostrand, Princeton, N.J. 1960. MR 22 \#5903.

6. B. Yood, Ideals in topological rings, Canad. J. Math. 16 (1964), 28-45. MR 28 \#1505.

7. - On algebras which are pre-Hilbert spaces, Duke Math. J. 36 (1969), 261-272. MR 41 \#4242.

Department of Mathematics, McMaster University, Hamilton, Ontario, CANADA 